Bull. Austral. Math. Soc.

VoL. 52 (1995) [475-486]

\title{
ESTIMATES BY POLYNOMIALS
}

\author{
R.M. ARon, Y.S. Chor and J.G. Llavona
}

Consider the following possible properties which a Banach space $X$ may have: (P): If $\left(x_{j}\right)$ and $\left(y_{j}\right)$ are bounded sequences in $X$ such that for all $n \geqslant 1$ and for every continuous n-homogeneous polynomial $P$ on $X, P\left(x_{j}\right)-P\left(y_{j}\right) \rightarrow 0$, then $Q\left(x_{j}-y_{j}\right) \rightarrow 0$ for all $m \geqslant 1$ and for every continuous m-homogeneous polynomial $Q$ on $X$.

(RP): If $\left(x_{j}\right)$ and $\left(y_{j}\right)$ are bounded sequences in $X$ such that for all $n \geqslant 1$ and for every continuous $n$-homogeneous polynomial $P$ on $X, P\left(x_{j}-y_{j}\right) \rightarrow 0$, then $Q\left(x_{j}\right)-Q\left(y_{j}\right) \rightarrow 0$ for all $m \geqslant 1$ and for every continuous m-homogeneous polynomial $Q$ on $X$. We study properties $(P)$ and $(R P)$ and their relation with the Schur property, Dunford-Pettis property, $\Lambda$, and others. Several applications of these properties are given.

During the past few years, interest has grown in the relation between a Banach space $X$ and spaces of polynomials on $X$. Thus, for example, Carne, Cole, and Gamelin [4] examined the relation of weak polynomial continuity on a Banach space, in what they called $\Lambda$-spaces, and tightness of certain Banach algebras of bounded analytic functions on the ball of a Banach space. Farmer [12] examined the relationship between upper and lower estimates on a Banach space $X$ and reflexivity of the Banach space of $n$ homogeneous polynomials on $X$, and Farmer and Johnson [13] studied polynomial versions of the Dunford-Pettis and Schur properties, relating them to the type of a Banach space. Earlier, Davie and Gamelin [7] had proved a strengthened form of Goldstine's theorem for weak polynomial density of $\mathrm{Ball}_{X}$ in Ball $\mathrm{X}^{* *}$ and, almost fifteen years ago, Ryan [20] had shown that the Dunford Pettis property is equivalent to the polynomial Dunford Pettis property.

The original motivation for this article was the following question:

Suppose that $X$ and $Y$ are real or complex Banach spaces with closed unit balls $B_{X}$ and $B_{Y}$ respectively, and let $g: B_{X} \rightarrow B_{Y}$ be a function which satisfies property (*), below:

(*) For any polynomial $P: Y \rightarrow \mathbf{K}=\mathbf{R}$ or $\mathbf{C}$, the composition $P \circ g: B_{X} \rightarrow$ $\mathrm{K}$ is uniformly continuous.

Received 27th February, 1995

Research for the first author was supported in part by NSF grant INT-9023951. For the second author research was supported by KOSEF and GARC-KOSEF and for the third author research was supported in part by DGICYT Grant PB90-0044. We acknowledge several helpful conversations with P. Cembranos, J. Mendoza, and P. and E. Saab.

Copyright Clearance Centre, Inc. Serial-fee code: 0004-9729/95 \$A2.00+0.00. 


\section{Is $g$ necessarily uniformly continuous?}

The natural approach to this question is to take two arbitrary sequences $\left(x_{n}\right)$ and $\left(y_{n}\right)$ in $B_{X}$ such that $\left\|x_{n}-y_{n}\right\| \rightarrow 0$, and to ask if the image sequences in $B_{Y}$ satisfy $\left\|g\left(x_{n}\right)-g\left(y_{n}\right)\right\| \rightarrow 0$. It is clear that by $(*)$, our problem is really to determine when the following condition is satisfied:

(**) Given two bounded sequences $\left(u_{n}\right)$ and $\left(v_{n}\right)$ in $Y$, if $\left|P\left(u_{n}\right)-P\left(v_{n}\right)\right| \rightarrow$ 0 for every continuous polynomial $P$ on $Y$, it follows that $\left\|u_{n}-v_{n}\right\| \rightarrow 0$.

We introduce here the following condition which, as we shall see, is strictly weaker than $(* *)$ :

Property $(P)$. Given two bounded sequences $\left(u_{j}\right)$ and $\left(v_{j}\right)$ in $Y$, if for every $n \geqslant 1$ and every $P \in \mathcal{P}\left({ }^{n} Y\right),\left|P\left(u_{j}\right)-P\left(v_{j}\right)\right| \rightarrow 0$, it follows that $\left|Q\left(u_{j}-v_{j}\right)\right| \rightarrow 0$ for every $m \geqslant 1$ and every $Q \in \mathcal{P}\left({ }^{m} Y\right)$.

(Here, $\mathcal{P}\left({ }^{n} Y\right)$ denotes the continuous $n$-homogeneous scalar-valued polynomials on $Y$. This space and related concepts will be reviewed at the beginning of Section 1.) In this article, we study property $(P)$ and its relation to other properties of a Banach space (such as the Dunford-Pettis property [8] or the $\Lambda$-property [4]). In addition, in our study of property $(P)$, we were led to the following reciprocal property, which is also studied in this paper:

"Reciprocal" Property $(R P)$. Given two bounded sequences $\left(u_{j}\right)$ and $\left(v_{j}\right)$ in $Y$, if for every $n \geqslant 1$ and every continuous $P \in \mathcal{P}\left({ }^{n} Y\right),\left|P\left(u_{j}-v_{j}\right)\right| \rightarrow 0$, it follows that $\left|Q\left(u_{j}\right)-Q\left(v_{j}\right)\right| \rightarrow 0$ for every $m \geqslant 1$ and every $Q \in \mathcal{P}\left({ }^{m} Y\right)$.

We study property $(P)$ in Section 1 . Property $(R P)$ has been used in examining versions of Arens regularity for multilinear mappings [2], and in Section 2, we give examples of Banach spaces which have, and which don't have, this property. We give several applications and discuss the relation between these and other known properties. However, we remark that the basic question of whether every Banach space has property $(P)$ remains open.

\section{Property $(P)$}

We begin by recalling some relevant definitions and reviewing several properties of Banach spaces related to continuity of polynomials. $X$ and $Y$ are real or complex Banach spaces with closed unit balls $B_{X}$ and $B_{Y}$ respectively. For $n=0,1, \ldots$, the space $\mathcal{P}\left({ }^{n} X, Y\right)$ of continuous $n$-homogeneous polynomials $P: X \rightarrow Y$ consists of all functions $P$ of the form $P(x)=A(x, \ldots, x)$, where $A: X \times \cdots \times X \rightarrow Y$ is a continuous $n$-linear mapping. $\|P\| \equiv \sup \left\{\|P(x)\|: x \in B_{X}\right\}$. The space $P(X, Y)$ is the algebraic direct sum of the spaces $\mathcal{P}\left({ }^{n} X, Y\right), n=0,1,2, \ldots$ We use $\mathcal{P}(X)$ and $\mathcal{P}\left({ }^{n} X\right)$ to denote 
$\mathcal{P}(X, \mathbf{K})$ and $\mathcal{P}\left({ }^{n} X, \mathbf{K}\right)$, respectively. In [4], the following type of space was introduced and studied:

Definition 1.1: [4] A Banach space $X$ is a $\Lambda$-space if for any sequence $\left(u_{k}\right) \subset X$ such that $\left(P\left(u_{k}\right)\right) \rightarrow 0$ for all $P \in \mathcal{P}\left({ }^{n} X\right)(n \geqslant 1)$ as $k \rightarrow \infty$, it follows that $\left\|u_{k}\right\| \rightarrow 0$.

The second author and Kim [6] have obtained connections between $\Lambda$-spaces and spaces having properties $(P)$ and $(R P)$. We list below several basic examples and properties of $\Lambda$-spaces.

\section{PROPOSITION 1.2.}

1. [14] Every super-reflexive Banach space is a $\Lambda$-space. In particular, every $\mathcal{L}^{p}$-space is a $\Lambda$-space, for $1<p<\infty$.

2. [4] Closed subspaces of $\Lambda$-spaces and Schur spaces are $\Lambda$-spaces.

3. . [4] $L_{1}[0,1], c_{0}$, and $\ell_{\infty}$ are not $\Lambda$-spaces.

4. [13] Every Banach space with non-trivial type is a $\Lambda$-space.

Definition 1.3: A function $f: B_{X} \rightarrow \mathrm{K}$ is said to be $\mathcal{P}$-continuous if for every $\varepsilon>0$, there are $\delta>0$ and a finite set $\left\{P_{1}, \ldots, P_{n}\right\} \subset \mathcal{P}(X)$ such that if $x, y \in B_{X}$ satisfy $\left|P_{j}(x-y)\right|<\delta,(j=1, \ldots, n)$, then $|f(x)-f(y)|<\varepsilon$.

In [3], the authors study the special case of $\mathcal{P}$-continuity, when the polynomials are restricted to being 1-homogeneous (that is, continuous linear forms), obtaining the space $C_{w u}\left(B_{X}\right)$ of functions which are weakly uniformly continuous on the ball of $X$. For certain Banach spaces, such as $c_{0}$, every $\mathcal{P}$-continuous function is automatically weakly uniformly continuous, but in general these two spaces are different. For example, $\sum_{j=1}^{\infty} x_{j}^{2}$ is clearly $\mathcal{P}$-continuous on $\ell_{2}$ although it cannot be weakly uniformly continuous on $B_{\ell_{2}}$. On the other hand, every $\mathcal{P}$-continuous function is uniformly continuous. For many Banach spaces such as $\ell_{2}$, uniform and $\mathcal{P}$-continuity coincide; on the other hand, the norm on $c_{0}$ is not $\mathcal{P}$-continuous. We do not know of a characterisation of those Banach spaces $X$ for which uniform and $\mathcal{P}$-continuity coincide.

We shall appeal to the following useful result of R.A. Ryan.

Proposition 1.4. [20] The Dunford-Pettis and the polynomial DunfordPettis properties are equivalent. That is, if $X$ has the Dunford-Pettis property, if $Y$ is an arbitrary Banach space, and if $P: X \rightarrow Y$ is a polynomial such that $P\left(B_{X}\right)$ is relatively weakly compact in $Y$, then $\left(P\left(x_{n}\right)\right)$ is norm Cauchy in $Y$ for every weakly Cauchy sequence $\left(x_{n}\right)$ in $X$.

This result can be used to give a straightforward proof of the result that a Banach space $X$ is a Schur space if and only if $X$ is a $\Lambda$-space and has the Dunford-Pettis property [4]. Indeed, let $\left(x_{j}\right)$ be a weakly null sequence in $X$. By Proposition 1.4, it follows that $\left(P\left(x_{j}\right)\right)$ is a norm null sequence of scalars, and so since $X$ is a $\Lambda$-space it 
follows that $\left\|x_{j}\right\| \rightarrow 0$.

We begin with the following easy facts about property $(P)$.

Proposition 1.5 .

(i) Every Banach space with the Dunford-Pettis property has ( $P$ ).

(ii) Let $g: B_{X} \rightarrow B_{Y}$ be a function between the closed unit balls of Banach spaces $X$ and $Y$, such that property $\left({ }^{*}\right)$ holds. If $Y$ is a $\Lambda$-space with property $(P)$, then $g$ is uniformly continuous.

Proof: (i) If $\left(u_{n}\right)$ and $\left(v_{n}\right)$ satisfy the conditions in 1.5 , then clearly $\left(u_{n}-v_{n}\right) \rightarrow$ 0 weakly. Consequently, since every scalar-valued polynomial $Q$ is automatically weakly compact, an application of 1.4 completes the proof. The proof of (ii) is straightforward.

In fact, all that is used in the proof of $1.5(i)$ is the fact that if a sequence $\left(x_{j}\right) \rightarrow 0$ weakly in $X$, then $\left(Q\left(x_{j}\right)\right) \rightarrow 0$ for every $Q \in \mathcal{P}\left({ }^{n} X\right)$ and every $n$. In particular, if every continuous scalar-valued polynomial on $X$ is weakly sequentially continuous at the origin, then $X$ has property $(P)$. Thus, for example, the original Tsirelson space $T^{*}$ has $(P)[1]$.

If $g: B_{c_{0}} \rightarrow B_{c_{0}}$ is defined by $g\left(\left(x_{n}\right)\right)=\left(x_{n}^{n}\right)$, then $g$ is not uniformly continuous since $\left\|g\left(e_{n}\right)-g\left([1-(1 / n)] e_{n}\right)\right\| \rightarrow 1-e^{-1}>0$. However, by the LittlewoodBogdanowicz-Pelczynski theorem (see, for example, [17]), $P \circ g$ is uniformly continuous for every continuous polynomial $P$ on $c_{0}$. Although the spaces $L_{1}[0,1], c_{0}$, and $\ell_{\infty}$ satisfy property $(P)$, they do not satisfy condition $(* *)$.

In order to prove that a space containing some $\ell_{p}, 1<p<\infty$, is a $\Lambda$-space, it is sufficient to only make use of polynomials of degree $[p]$. However, the following simple example shows that something more is required to prove that a space has property $(P)$. Consider the two sequences $\left(e_{j}\right)$ and $\left(-e_{j}\right)$ in $\ell_{2}$. For this pair of sequences, it is clear $\left(e_{j}-\left(-e_{j}\right)\right) \rightarrow 0$ weakly and that $P\left(e_{j}\right)-P\left(-e_{j}\right) \rightarrow 0$ for all 2-homogeneous continuous polynomials $P$ on $\ell_{2}$. The "clue" here is to allow polynomials of an odd degree greater than $p$; in this case, the 3 -homogeneous polynomial $P(x)=\sum_{n=0}^{\infty} x_{n}^{3}$ serves to show that $P\left(e_{j}\right)-P\left(-e_{j}\right) \nrightarrow 0$.

Castillo and Sánchez define in [5] a Banach space $X$ to be in the class $W_{p}(1<p$ $<\infty)$ when each bounded sequence $\left(x_{j}\right)$ in $X$ admits a weakly-p-convergent subsequence $\left(x_{j_{k}}\right)$; that is, there is $x \in X$ such that $\sum_{k=1}^{\infty}\left|x^{*}\left(x_{j_{k}}-x\right)\right|^{p}<+\infty$ for all $x^{*} \in X^{*}$. They showed that every super-reflexive Banach space is in the class $W_{p}$ for some $p, 1<p<\infty$. We use this fact in proving Theorem 1.6.

THEOREM 1.6. If $X$ is a Banach space whose dual $X^{*}$ is in $W_{p}$ for some $p \in$ $(1, \infty)$, then $X$ has property $(P)$. 
Proof: Let $\left(x_{j}\right)$ and $\left(y_{j}\right)$ be bounded sequences in $X$ such that $\left|P\left(x_{j}\right)-P\left(y_{j}\right)\right| \rightarrow$ 0 for every $P \in \mathcal{P}\left({ }^{n} X\right), n \geqslant 1$. We claim that $\left\|x_{j}-y_{j}\right\| \rightarrow 0$. Otherwise there exist some $\varepsilon>0$ and subsequences of $\left(x_{j}\right)$ and $\left(y_{j}\right)$, which we shall still denote by $\left(x_{j}\right)$ and $\left(y_{j}\right)$, such that $\left\|x_{j}-y_{j}\right\| \geqslant \varepsilon$ for every $j$. Since $\left(x_{j}-y_{j}\right)$ goes to 0 weakly, by passing to a subsequence, $\left(x_{j}-y_{j}\right)$ can be considered as a basic sequence in $X$ (see for example, [9]). Let $z_{j}=x_{j}-y_{j}$ and $Z$ be the closed subspace of $X$ spanned by $\left(z_{j}\right)$. Let $\left(z_{j}^{*}\right)$ be the corresponding bounded sequence of coefficient functionals associated to $\left(z_{j}\right)$. By the Hahn-Banach theorem each $z_{j}^{*}$ can be extended to a functional $z_{j}^{*} \in X^{*}$ with the same norm, and hence $\left(z_{j}^{*}\right)$ is a bounded sequence in $X^{*}$. Since $X^{*}$ is in $W_{p}$, it follows from [5] that there exist an $x^{*} \in X^{*}$ and a subsequence of $\left(z_{j}^{*}\right)$, still denoted by $\left(z_{j}^{*}\right)$, such that

$$
\sum_{j=1}^{\infty}\left|\left(z_{j}^{*}-x^{*}\right)(x)\right|^{p}<\infty \quad(x \in X) .
$$

For any $z \in Z,\left(z_{j}^{*}-x^{*}\right)(z) \rightarrow 0$ and $z_{j}^{*}(z) \rightarrow 0$. This implies $x^{*}(z)=0$ for every $z \in Z$. Hence we obtain

$$
\left(z_{j}^{*}-x^{*}\right)\left(x_{\ell}\right)-\left(z_{j}^{*}-x^{*}\right)\left(y_{\ell}\right)=z_{j}^{*}\left(z_{\ell}\right)=\delta_{j \ell},
$$

where $\delta_{j \ell}$ is the Kronecker delta.

By taking subsequences, we may assume that $\left(z_{j}^{*}-x^{*}\right)\left(x_{j}\right)$ and $\left(z_{j}^{*}-x^{*}\right)\left(y_{j}\right)$ converge to, say, $a$ and $b$ respectively. Clearly, $a-b=1$. Choose $N>p$ such that $\left|a^{N}-b^{N}\right|=R>0$, and define

$$
P(x)=\sum_{j=1}^{\infty}\left[\left(z_{j}^{*}-x^{*}\right)(x)\right]^{N} \quad(x \in X) .
$$

It is easy to see that $P \in \mathcal{P}\left({ }^{N} X\right)$. Moreover, for each $\ell$ we have

$$
\left|P\left(x_{\ell}\right)-P\left(y_{\ell}\right)\right|=\left|\left(z_{\ell}^{*}-x^{*}\right)\left(x_{\ell}\right)^{N}-\left(z_{\ell}^{*}-x^{*}\right)\left(y_{\ell}\right)^{N}\right| \rightarrow R,
$$

which contradicts the original hypothesis.

Note that the dual of a super-reflexive space is also super-reflexive (see, for example, [10]). The proof of Theorem 1.6 shows that if $X$ is super-reflexive, then $X$ has property $(P)$. Since $L_{p}[0,1]$ is super-reflexive for $1<p<\infty$, every such $L_{p}[0,1]$ has property $(P)$.

Using [13] and a modification of the above argument, Choi and Kim [6] have shown that every space which has non-trivial type has property $(P)$. We remark that we have no example of any Banach space which does not have property $(P)$. Moreover, although 
it is trivial that every Banach space which has property (**) described above is a $\Lambda$ space, we do not know if the converse implication holds. However, every $\Lambda$-space which has property $(P)$ satisfies $(* *)$.

We conclude this section by commenting on a question of Petunin and Savkin [18], concerning an analytic version of a $\Lambda$-space. $X$ is said to be a holomorphic $\Lambda$-space if, whenever $\left(x_{n}\right) \subset X$ is a sequence such that $\left(f\left(x_{n}\right)\right) \rightarrow f(0)$ for every holomorphic function $f: X \rightarrow \mathbf{C}$, then $\left\|x_{n}\right\| \rightarrow 0$. Of course, every $\Lambda$-space is a holomorphic $\Lambda$-space. The converse is false since, for example, every separable Banach space is a holomorphic $\Lambda$-space [18]. The following shows that the not every Banach space is a holomorphic $\Lambda$-space.

EXAMPLE 1.7. $\ell_{\infty}$ is not a holomorphic $\Lambda$-space.

Proof: Let $\left(e_{n}\right)$ be the standard unit vector basis of $c_{0} \subset \ell_{\infty}$, and let $f \in H\left(\ell_{\infty}\right)$ with Taylor series representation $\sum_{j=0}^{\infty} P_{j}$. By a result of Josefson [15], $\left.f\right|_{c_{0}}$ is bounded on every bounded subset of $c_{0}$, and so if $\bar{B}$ denotes the closed unit ball of $c_{0},\left.f\right|_{\bar{B}}$ is the uniform limit of $\left.\sum_{j=0}^{\infty} P_{j}\right|_{\bar{B}}$. Since $P_{j}\left(e_{n}\right) \rightarrow 0$ as $n \rightarrow \infty$ for each $j=1,2, \ldots$, (see, for example, [17] or [20]), it follows easily that $\left(f\left(e_{n}\right)\right) \rightarrow f(0)$, which completes the proof.

\section{Property $(R P)$}

We begin with several elementary examples of spaces which have $(R P)$.

Proposition 2.1. 1. Every $\Lambda$-space has property $(R P)$.

2. $c_{0}$ has property $(R P)$.

ProOF: Both proofs are easy. Let $\left(u_{j}\right)$ and $\left(v_{j}\right)$ be bounded sequences in $X$ and suppose that $P\left(u_{j}-v_{j}\right) \rightarrow 0$ for every $P \in \mathcal{P}\left({ }^{n} X\right), n \geqslant 1$. If $X$ is a $\Lambda$-space, then $\left\|u_{j}-v_{j}\right\| \rightarrow 0$. Since every $Q \in \mathcal{P}\left({ }^{n} X\right), n \geqslant 1$, is uniformly continuous on bounded subsets of $X$, we get $Q\left(u_{j}\right)-Q\left(v_{j}\right) \rightarrow 0$. Also, since $\left(u_{j}-v_{j}\right) \rightarrow 0$ weakly in $c_{0}$, $\left|\phi^{n}\left(u_{j}\right)-\phi^{n}\left(v_{j}\right)\right| \rightarrow 0$ for every $\phi \in c_{0}^{*}$. The result follows from the fact that every continuous polynomial on $c_{0}$ can be approximated uniformly on bounded subsets of $c_{0}$ by finite type polynomials [17].

Another proof that $c_{0}$ has $(R P)$ follows.

THEOREM 2.2. If $X$ has the Dunford-Pettis property and $\ell_{1} \nrightarrow X$, then $X$ has property $(R P)$.

Proof: Suppose that $\left(x_{j}\right)$ and $\left(y_{j}\right)$ are bounded sequences in $X$ and that $P\left(x_{j}-y_{j}\right) \rightarrow 0$ for every $P \in \mathcal{P}\left({ }^{n} X\right), n \geqslant 1$. By Rosenthal's dichotomy [19], since 
$\ell_{1} \nrightarrow X$, there exist weakly Cauchy subsequences of $\left(x_{j}\right)$ and $\left(y_{j}\right)$, which we still denote by $\left(x_{j}\right)$ and $\left(y_{j}\right)$. Let $Q \in \mathcal{P}\left({ }^{n} X\right), n \geqslant 1$. Since $X$ has the Dunford-Pettis property, we have by Proposition 1.4 that $Q\left(x_{j}\right) \rightarrow \ell$ and $Q\left(y_{j}\right) \rightarrow m$ for some $\ell$ and $m$. The sequence $\left(x_{1}, y_{1}, x_{2}, y_{2}, \ldots\right)$, is also weakly Cauchy, since for each $\varphi \in X^{*}$,

$$
\left|\varphi\left(x_{j}-y_{k}\right)\right| \leqslant\left|\varphi\left(x_{j}-x_{k}\right)\right|+\left|\varphi\left(x_{k}-y_{k}\right)\right| \rightarrow 0
$$

as $j, k \rightarrow \infty$. Hence $Q\left(x_{1}\right), Q\left(y_{1}\right), Q\left(x_{2}\right), Q\left(y_{2}\right), \ldots$ converges and so $\ell=m$, which proves that $Q\left(x_{j}\right)-Q\left(y_{j}\right) \rightarrow 0$ for every $Q \in \mathcal{P}\left({ }^{n} X\right), n \geqslant 1$.

We shall show that $\ell_{\infty} \approx L_{\infty}[0,1], C[0,1]$, and $L_{1}[0,1]$ do not have property $(R P)$. Thus, the Dunford-Pettis property is not, by itself, a sufficient condition to yield $(R P)$.

LEMMA 2.3. Let $\left(I_{j}\right)$ be a sequence of pairwise disjoint subsets of $[0,1]$. Then $\left(\chi_{I_{j}}\right)$ converges to 0 weakly in $L_{\infty}[0,1]$.

PROOF: If $\left(\chi_{I_{j}}\right)$ does not converge to 0 weakly in $L_{\infty}[0,1]$, we may multiply $\chi_{I_{j}}$ by a suitable complex number having modulus 1 and so assume without loss of generality that $\varphi\left(\chi_{I_{j}}\right) \geqslant \varepsilon$ for some $\varphi \in\left(L_{\infty}[0,1]\right)^{*}$ of norm 1 and some $\varepsilon>0$. Let $f=\sum \chi_{I_{j}} \in L_{\infty}[0,1]$ with $\|f\|_{\infty}=1$. For each $n$

$$
\begin{aligned}
|\varphi(f)| & =\left|\varphi\left(\sum_{j=1}^{n} \chi_{I_{j}}+\sum_{j=n+1}^{\infty} \chi_{I_{j}}\right)\right| \\
& =\left|\sum_{j=1}^{n} \varphi\left(\chi_{I_{j}}\right)+\varphi\left(\sum_{j=n+1}^{\infty} \chi_{I_{j}}\right)\right| \\
& \geqslant n \varepsilon-\left|\varphi\left(\sum_{j=n+1}^{\infty} \chi_{I_{j}}\right)\right| \geqslant n \varepsilon-\|\varphi\|,
\end{aligned}
$$

which contradicts $|\varphi(f)| \leqslant 1$.

EXAMPLE 2.4. $L_{\infty}[0,1]$ does not have property $(R P)$.

Proof: Let $I_{j}=\left(1 / 2^{j}, 1 / 2^{j-1}\right)(j=1,2, \ldots)$ and let $\left(r_{j}(t)\right)_{j=1}^{\infty}$ be the Rademacher functions on $[0,1]$ defined by $r_{j}(t)=\operatorname{sign}\left(\sin 2^{j} \pi t\right)$. Define

$$
A: L_{\infty}[0,1] \times L_{\infty}[0,1] \times L_{\infty}[0,1] \rightarrow \mathbf{K}
$$

by

$$
A(f, g, h)=\sum_{j=1}^{\infty}\left(2^{j} \int_{I_{j}} f d x\right)\left\langle g, r_{j}\right\rangle\left\langle h, r_{j}\right\rangle
$$


where $\left\langle g, r_{j}\right\rangle=\int_{0}^{1} g r_{j} d x$. For $\|f\|_{\infty} \leqslant 1,\|g\|_{\infty} \leqslant 1,\|h\|_{\infty} \leqslant 1$, we have

$$
\begin{aligned}
|A(f, g, h)| & \leqslant \sum_{j=1}^{\infty}\left|\left\langle g, r_{j}\right\rangle\left\langle h, r_{j}\right\rangle\right| \\
& \leqslant\left(\sum_{j=1}^{\infty}\left|\left\langle g, r_{j}\right\rangle\right|^{2}\right)^{1 / 2}\left(\sum_{j=1}^{\infty}\left|\left\langle h, r_{j}\right\rangle\right|^{2}\right)^{1 / 2} \\
& \leqslant\|g\|_{2}\|h\|_{2} \leqslant\|g\|_{\infty}\|h\|_{\infty} \leqslant 1,
\end{aligned}
$$

with the second inequality coming from Bessel's inequality. From this it is easy to see that $A$ is a continuous 3-linear map. For each $j$, let $s_{j}=\chi_{I_{j}}, x_{j}=r_{j}$ and $y_{j}=$ $r_{j}+s_{j}$. By the Dunford-Pettis property of $L_{\infty}[0,1]$, Lemma 2.3, and Proposition 1.4, $P\left(x_{j}-y_{j}\right) \rightarrow 0$ for every $P \in \mathcal{P}\left({ }^{n} L_{\infty}[0,1]\right), n \geqslant 1$. Let $Q \in \mathcal{P}\left({ }^{3} L_{\infty}[0,1]\right)$ be defined by $Q(f)=A(f, f, f)$ for $f \in L_{\infty}[0,1]$. Since

$$
\int_{I_{j}} r_{n} d x= \begin{cases}0, & \text { if } 1 \leqslant j \leqslant n-1 \\ -\frac{1}{2^{j}}, & \text { if } j=n \\ \frac{1}{2^{j}}, & \text { if } j>n,\end{cases}
$$

we see that $Q\left(r_{n}\right)=-1$ for all $n$. Similarly, $Q\left(y_{n}\right) \equiv 0$, and so $Q\left(x_{n}\right)-Q\left(y_{n}\right) \nrightarrow 0$, which completes the proof.

It is worth noting that $Q\left(x_{j}\right)-Q\left(y_{j}\right) \rightarrow 0$ for every $Q \in \mathcal{P}\left({ }^{2} \ell_{\infty}\right)$. In fact, suppose that $\left(x_{i}\right)$ and $\left(y_{i}\right)$ are bounded sequences in $\ell_{\infty}$ such that $\left(x_{j}-y_{j}\right)$ converges to 0 weakly in $\ell_{\infty}$. Let $Q \in \mathcal{P}\left({ }^{2} \ell_{\infty}\right)$ and $A$ be the unique continuous symmetric 2-linear map associated with $Q$, via $Q(x)=A(x, x)$. We can regard $A$ as the bounded linear map $A: x \in \ell_{\infty} \rightarrow A(x,) \in \ell_{\infty}^{*}$, Since $c_{0} \leftrightarrow \ell_{\infty}^{*}$, it follows that $A: \ell_{\infty} \rightarrow \ell_{\infty}^{*}$ is weakly compact [16]. By the Dunford-Pettis property of $\ell_{\infty}$, we get $\left\|A\left(x_{j}-y_{j}\right)\right\| \rightarrow 0$ in $\ell_{\infty}^{*}$. Now

$$
\begin{aligned}
\left|Q\left(x_{j}\right)-Q\left(y_{j}\right)\right| & =\left|A\left(x_{j}, x_{j}\right)-A\left(y_{j}, y_{j}\right)\right| \\
& =\left|A\left(x_{j}-y_{j}, x_{j}\right)+A\left(x_{j}-y_{j}, y_{j}\right)\right| \\
& \leqslant\left\|A\left(x_{j}-y_{j}\right)\right\|\left\|x_{j}\right\|+\left\|A\left(x_{j}-y_{j}\right)\right\|\left\|y_{j}\right\| \\
& \leqslant M\left\|A\left(x_{j}-y_{j}\right)\right\| \rightarrow 0
\end{aligned}
$$

where $\left\|x_{j}\right\|,\left\|y_{n}\right\| \leqslant M$ for all $j$. On the other hand, $L\left({ }^{2} \ell_{\infty}\right) \approx\left(\ell_{\infty} \otimes_{\pi} \ell_{\infty}\right)^{*}$ contains a copy of $c_{0}$. (A sketch of the argument is as follows: $L\left({ }^{2} \ell_{\infty}\right) \cong L\left(L_{\infty}, L_{\infty}^{*}\right)$ contains an isometric copy of $L_{1} \widehat{\bigotimes}_{\varepsilon} L_{1}$. In turn, $L_{1} \widehat{\bigotimes}_{e} L_{1}$ contains the weakly unconditionally convergent series $\sum_{j} r_{j} \otimes r_{j}$, which is not unconditionally convergent.)

The Rademacher functions play a similar role in the following argument, that $C[0,1]$ also fails to have property $(R P)$. 
EXAMPLE 2.5. $C[0,1]$ does not have property $(R P)$.

Proof: Define $A: C[0,1] \times C[0,1] \times C[0,1] \rightarrow \mathbf{K}$ by

$$
A(f, g, h)=\sum_{j=1}^{\infty} f\left(\frac{1}{2^{j}}\right)\left\langle g, r_{j}\right\rangle\left\langle h, r_{j}\right\rangle
$$

As in the previous example, $A$ is easily seen to be a continuous 3 -linear map. For $n \geqslant 2$, let

$$
f_{n}(t)= \begin{cases}0 & 0 \leqslant t \leqslant \frac{1}{2^{n+1}} \\ 2^{n+1}\left(t-\frac{1}{2^{n+1}}\right) & \frac{1}{2^{n+1}} \leqslant t \leqslant \frac{1}{2^{n}} \\ -2^{n+1}\left(t-\frac{3}{2^{n+1}}\right) & \frac{1}{2^{n}} \leqslant t \leqslant \frac{3}{2^{n+1}} \\ 0 & \frac{3}{2^{n+1}} \leqslant t \leqslant \frac{1}{2}\end{cases}
$$

on $[0,1 / 2]$ and for $1 / 2+(j-1) / 2^{n+1} \leqslant t \leqslant 1 / 2+j / 2^{n+1}\left(j=1,2, \ldots, 2^{n}\right)$ let

$$
f_{n}(t)= \begin{cases}-2^{n+1}\left(t-\left(\frac{1}{2}+\frac{j}{2^{n+1}}\right)\right) & j \equiv 0(\bmod 4) \\ -2^{n+1}\left(t-\left(\frac{1}{2}+\frac{j-1}{2^{n+1}}\right)\right) & j \equiv 1(\bmod 4) \\ 2^{n+1}\left(t-\left(\frac{1}{2}+\frac{j}{2^{n+1}}\right)\right) & j \equiv 2(\bmod 4) \\ 2^{n+1}\left(t-\left(\frac{1}{2}+\frac{j-1}{2^{n+1}}\right)\right) & j \equiv 3(\bmod 4) .\end{cases}
$$

Let $g_{n}=-f_{n} \chi_{[0,1 / 2]}+f_{n} \chi_{[1 / 2,1]},(n \geqslant 2)$. Then $\left(f_{n}-g_{n}\right)=\left(2 f_{n} \chi_{[0,1 / 2]}\right)$ is a bounded sequence in $C[0,1]$ which is pointwise convergent to 0 . By [11] $\left(f_{n}-g_{n}\right)$ converges to 0 weakly in $C[0,1]$ and so $P\left(f_{n}-g_{n}\right) \rightarrow 0$ for every $P \in \mathcal{P}\left({ }^{m} C[0,1]\right), m \geqslant 1$, by the Dunford-Pettis property of $C[0,1]$. Let $Q=\widehat{A} \in \mathcal{P}\left({ }^{3} C[0,1]\right)$, where $\widehat{A}(f)=A(f, f, f)$ for $f \in C[0,1]$. Now

$$
\begin{aligned}
Q\left(f_{n}\right)-Q\left(g_{n}\right) & =f_{n}\left(\frac{1}{2^{n}}\right)\left\langle f_{n}, r_{n}\right\rangle^{2}-g_{n}\left(\frac{1}{2^{n}}\right)\left\langle g_{n}, r_{n}\right\rangle^{2} \\
& =2\left\langle f_{n}, r_{n}\right\rangle^{2} \\
& =2 \cdot\left(2^{n-1} \times \frac{1}{2^{n+1}}\right)^{2}=\frac{1}{8}
\end{aligned}
$$

for all $n \geqslant 2$, which completes the proof. 
EXAMPLE 2.6. $L_{1}[0,2 \pi]$ does not have property $(R P)$.

ProOF: Let $I_{j}=\left((2 \pi) / 2^{j},(2 \pi) / 2^{j-1}\right)(j=1,2, \ldots)$ and let $\left(r_{j}(t)\right)_{j=1}^{\infty}$ be the Rademacher functions on $[0,2 \pi]$ defined by $r_{j}(t)=\operatorname{sign}\left(\sin 2^{j-1} t\right)$. Define $A$ : $L_{1}[0,2 \pi] \times L_{1}[0,2 \pi] \rightarrow \mathbf{K}$ by

$$
A(f, g)=\sum_{j=1}^{\infty}\left(\int_{I_{j}} f r_{j} d x\right)\left\langle g, r_{j}\right\rangle .
$$

For $\|f\|_{1},\|g\|_{1} \leqslant 1$, we get $|A(f, g)| \leqslant\|f\|_{1}\|g\|_{1}$ and so $A$ is a continuous bilinear map. For $j \geqslant 2$, let

$$
\begin{aligned}
f_{j}= & -\sin 2^{j-1} t+2 \chi_{\left[0,(2 \pi) / 2^{j}\right]} \sin 2^{j-1} t \\
& +2 \chi_{\left[2 \pi-(2 \pi) / 2^{j}, 2 \pi\right]} \sin 2^{j-1} t \\
& +2^{j} /(2 \pi) \chi_{I_{j}}
\end{aligned}
$$

and

$$
\begin{aligned}
g_{j}= & -2 \sin 2^{j-1} t+2 \chi_{\left[0,(2 \pi) / 2^{j}\right]} \sin 2^{j-1} t \\
& +2 \chi_{\left[2 \pi-(2 \pi) / 2^{j}, 2 \pi\right]} \sin 2^{j-1} t \\
& +\frac{2^{j}}{2 \pi} \chi_{I_{j}} \cdot
\end{aligned}
$$

Now, $f_{j}-g_{j}=\sin 2^{j-1} t$ converges to 0 weakly in $L_{1}[0,2 \pi]$ and so $P\left(f_{j}-g_{j}\right) \rightarrow 0$ for every $P \in \mathcal{P}\left({ }^{n} L_{1}[0,2 \pi]\right), n \geqslant 1$, by the Dunford-Pettis property of $L_{1}[0,2 \pi]$. Let $Q=\widehat{A} \in \mathcal{P}\left({ }^{2} L_{1}[0,2 \pi]\right)$, where $\widehat{A}(f)=A(f, f)$ for $f \in L_{1}[0,2 \pi]$. Note that

1. $\int_{I_{j}} f_{n} r_{j} d x=\int_{I_{j}} g_{n} r_{j} d x=0$ for $2 \leqslant j<n$,

2. $\left\langle f_{n}, r_{j}\right\rangle=\left\langle g_{n}, r_{j}\right\rangle=0$ for $j>n$,

Using these facts, we can calculate

$$
\begin{aligned}
Q\left(f_{n}\right) & =\left(\int_{I_{1}} f_{n} r_{1} d x\right)\left\langle f_{n}, r_{1}\right\rangle+\left(\int_{I_{n}} f_{n} r_{n} d x\right)\left\langle f_{n}, r_{n}\right\rangle \\
& =\left(2 \cdot 2^{2-n}\right)\left(1+4 \cdot 2^{2-n}\right)+\left(-1-2^{2-n}\right)\left[-2^{2-n}\left(2^{n}-4\right)-1\right]
\end{aligned}
$$

and

$$
\begin{aligned}
Q\left(g_{n}\right) & =\left(\int_{I_{1}} g_{n} r_{1} d x\right)\left\langle g_{n}, r_{1}\right\rangle+\left(\int_{I_{n}} g_{n} r_{n} d x\right)\left\langle g_{n}, r_{n}\right\rangle \\
& =2^{3-n}\left(1+2 \cdot 2^{3-n}\right)+\left(-1-2^{3-n}\right)\left[-2^{3-n}\left(2^{n}-2\right)-1\right]
\end{aligned}
$$

for each $n, n \geqslant 2$. Hence $Q\left(f_{n}\right)-Q\left(g_{n}\right)=-4-52 \cdot\left(2^{-n}\right)+2^{6} \cdot\left(2^{-2 n}\right) \rightarrow-4$, which completes the proof.

Finally, if in a Banach space, every (norm) continuous polynomial is $P$-continuous in the sense of Definition 1.3, then property $(R P)$ holds. We remark that in each of the above examples, polynomials have been constructed which are not $P$-continuous. 


\section{REFERENCES}

[1] R. Alencar, R.M. Aron and S. Dineen, 'A reflexive space of holomorphic functions in infinitely many variables', Proc. Amer. Math. Soc. 90 (1984), 407-411.

[2] R.M. Aron and P. Galindo, 'Weakly compact multilinear mappings', (preprint).

[3] R.M. Aron and J.B. Prolla, 'Polynomial approximation of differentiable functions on Banach spaces', J. Reine Agnew. Math. 313 (1980), 195-216.

[4] T. Carne, B. Cole and T. Gamelin, 'A uniform algebra of analytic functions on a Banach space', Trans. Amer. Math. Soc. 314 (1989), 639-659.

[5] J.F. Castillo and C. Sánchez, 'Weakly-p-compact, p-Banach-Saks and super-reflexive Banach spaces', J. Math. Anal. Appl. 185 (1994), 256-261.

[6] Y.S. Choi and S.G. Kim, 'Polynomial properties of Banach spaces', J. Math. Anal. Appl. 190 (1995), 203-210.

[7] A.M. Davie and T.W. Gamelin, 'A theorem on polynomial-star approximation', Proc. Amer. Math. Soc. 106 (1989), 351-358.

[8] J. Diestel, 'A survey of results related to the Dunford-Pettis property', in Contemp. Math. 2 (Amer. Math. Soc., Providence, R.I., 1980), pp. 15-60.

[9] J. Diestel, Geometry of Banach spaces, Lecture Notes in Mathematics 485 (Springer-Verlag, Berlin, Heidelberg, New York, 1975).

[10] D. Van Dulst, Reflexive and super-reflexive spaces, Math. Centre Tracts 102 (Amsterdam, 1982).

[11] N. Dunford and J.T. Schwartz, Linear Operators, Part I, General Theory (J. Wiley, New York, 1964).

[12] J.D. Farmer, 'Polynomial reflexivity in Banach spaces', Israel J. Math. 87 (1994), 257-273.

[13] J.D. Farmer and W.B. Johnson, 'Polynomial Schur and polynomial Dunford-Pettis properties', in Proc. Intern. Research Workshop on Banach Space Theory (Mérida, Venezuela), (W.B. Johnson and B. L. Lin, Editors) (Amer. Math. Soc., Providence, RI, 1993), pp. 95-105.

[14] J.A. Jaramillo and A. Prieto, 'Weak-polynomial convergence on a Banach space', Proc. Amer. Math. Soc. 118 (1993), 463-468.

[15] B. Josefson, 'Bounding subsets of $\ell^{\infty}(A)$ ', J. Math. Pures Appl. 57 (1978), 397-421.

[16] A. Grothendieck, 'Sur les applications linéaires faiblement compactes d'espaces du type $C(K)^{\prime}$, Canad. J. Math. 5 (1953), 129-173.

[17] A. Pelczynski, 'A property of multilinear operations', Studia Math. 16 (1957), 173-182.

[18] Y.I. Petunin and V.I. Savkin, 'Convergence generated by analytic functions', Ukranian. Math. J. 40 (1988), 676-679.

[19] H.P. Rosenthal, 'Some recent discoveries in the isomorphic theory of Banach spaces', Bull. Amer. Math. Soc. 84 (1980), 803-831.

[20] R.A. Ryan, 'Dunford-Pettis properties', Bull. Acad. Polon. Sci. Math. 27 (1979), 373-379. 
Department of Mathematics

Kent State University

Kent Oh 44242

United States of America

Departamento de Análisis Matemático

Universidad Complutense de Madrid

28040 Madrid

Spain
Department of Mathematics

Pohang University of Science and Technology

Pohang

Korea 790 